\title{
Nanofibrillar hydrogel recapitulates changes occurring in the fibrotic extracellular matrix
}

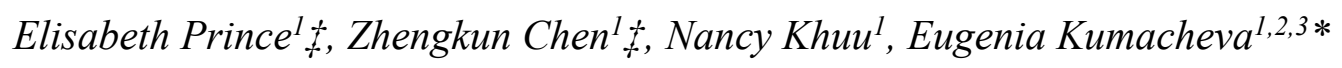

${ }^{1}$ Department of Chemistry, University of Toronto, 80 Saint George Street, Toronto, Ontario, Canada, M5S 3H6.

${ }^{2}$ Department of Chemical Engineering and Applied Chemistry, University of Toronto, 200 College Street, Toronto, Ontario, Canada, M5S 3E5.

${ }^{3}$ Institute of Biomaterials and Biomedical Engineering, University of Toronto, 4 Taddle Creek Road, Toronto, Ontario, Canada, M5S 3G9.

\section{Section S1. Experimental design for determining EKGel permeability}

Figure S1a illustrates the experimental set up for measurements of EKGel Darcy permeability. The EKGel with varying compositions was formed in a $3 \mathrm{~mm} \times 3 \mathrm{~mm} \times 13.7 \mathrm{~mm}$ (width $\times$ height $\times$ length) polydimethylsiloxane (PDMS) chamber. The chamber was connected to inlet and outlet reservoirs filled with HBSS by perfluoroalkoxyalkane tubing (IDEX Health \& Science). The inlet reservoir was placed higher than the outlet reservoir, resulting in a hydrostatic pressure drop $(\Delta \mathrm{P})$ across the hydrogel. The volumetric flow rate of the liquid through the hydrogel was determined by measuring the change in the mass of the outlet reservoir over time. 
To ensure that the EKG structure was not disrupted due to applied pressure, $\Delta \mathrm{P}$, we measured the volumetric flow rate, $\mathrm{Q}_{\mathrm{p}}$, as a function of $\Delta \mathrm{P}$. Figure $\mathrm{S} 1 \mathrm{~b}$ shows that for EKGel with $C_{\mathrm{CNC}}=1.0 \mathrm{wt} \%$ and $C_{\mathrm{gel}}=2 \mathrm{wt} \%$, the value of $\mathrm{Q}_{\mathrm{p}}$ increased linearly with $\Delta \mathrm{P}$ in accordance with Darcy's law. ${ }^{1}$ The Darcy permeability coefficient $\left(\mathrm{K}_{\mathrm{s}}\right)$ was determined as

$$
K_{S}=\frac{\eta L Q_{p}}{A \Delta P}
$$

where $L$ is the hydrogel length $(13.7 \mathrm{~mm}), \Delta \mathrm{P}$ is the pressure difference across the hydrogel calculated from the difference in height between the inlet at the outlet, $\eta$ is the viscosity of HBSS solution (taken as $1.002 \mathrm{cP}$ ), and $A$ is the cross-sectional area of the hydrogel $\left(9 \mathrm{~mm}^{2}\right)$.

The slope of the plot of $\mathrm{Q}_{\mathrm{p}}$ vs. $\Delta \mathrm{P}$, that is, $\frac{K_{s} L_{p} \eta}{A}$, should theoretically be equal to $1.2 \times 10^{-9} \frac{\mathrm{mL}}{\mathrm{s} \times \mathrm{Pa}}$, where $K_{\mathrm{s}}$ is the Darcy Permeability $\left(1.9 \times 10^{-11} \mathrm{~cm}^{2}\right.$ for $C_{\mathrm{CNC}}=1.0 \mathrm{wt} \%$ and $C_{\mathrm{gel}}=2 \mathrm{wt} \%$ ). Indeed, linear regression analysis shows that the slope of $\mathrm{Q}_{\mathrm{p}} \mathrm{vs} . \Delta \mathrm{P}$ is close to the theoretical value. The slope in Figure $\mathrm{S} 1 \mathrm{~b}$ is $1.1 \times 10^{-9} \frac{\mathrm{mL}}{\mathrm{s} \times \mathrm{Pa}}$ with an $\mathrm{R}^{2}=0.93$.
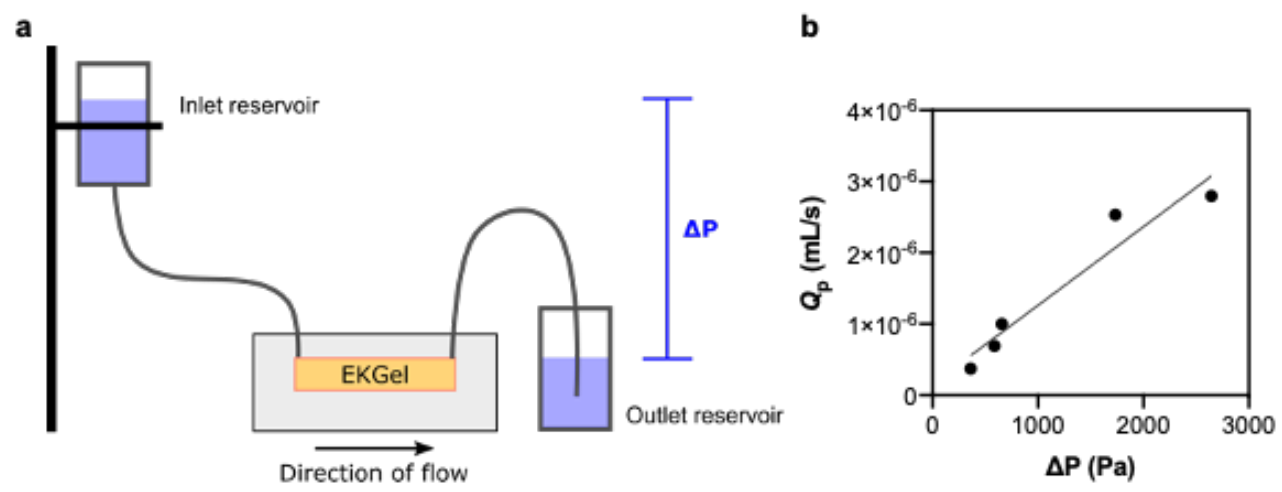

Figure S1. Experiment for measurements of Darcy permeability. (a) Experimental set-up used to determine EKGel Darcy permeability. (b) Linear dependance of volumetric flow rate, $Q_{\mathrm{p}}$, on the applied pressure drop, DP, for EKGel with $C_{\mathrm{CNC}}=1.0 \mathrm{wt} \%$, and $C_{\mathrm{gel}}=2 \mathrm{wt} \%$. In (b) the slope of the plot of $Q_{\mathrm{p}} v s$. DP is equal to $1.1 \times 10^{-9} \frac{\mathrm{mL}}{s \times P a}, \mathrm{R}^{2}=0.93$. 


\section{Section S2: Construction of the calibration curve for the determination of the concentration}

\section{of unreacted amines in EKGel}

In order to determine the number of imine crosslinks in EKGel, we characterized the concentration of free amine groups in EKGel (that is, those that were not consumed by crosslinking with CNCs) by using the 2,4,6-trinitrobenzylsulfonic acid (TNBS) assay. To calculate amine group concentration, we constructed a calibration graph. Briefly, solutions of glycine with concentrations in the range from 0.1 to $5 \mathrm{mM}$ in HBSS were prepared and plated in a 96 well plate $(50 \mu \mathrm{L}$ per well). Next, $100 \mu \mathrm{L}$ of $0.5 \mathrm{w} / \mathrm{v} \%$ TNBS in $0.1 \mathrm{M}$ sodium carbonate buffer ( $\mathrm{pH} 9$ ) was added to each well. The well plate was incubated for 2 hours at $37^{\circ} \mathrm{C}$, and then the absorbance at $500 \mathrm{~nm}$ was determined with a CLARIOStar plate reader. Figure S2 shows the calibration curve that was used to determine the concentration of imine crosslinks (see Figures 2e and 3e, main text).

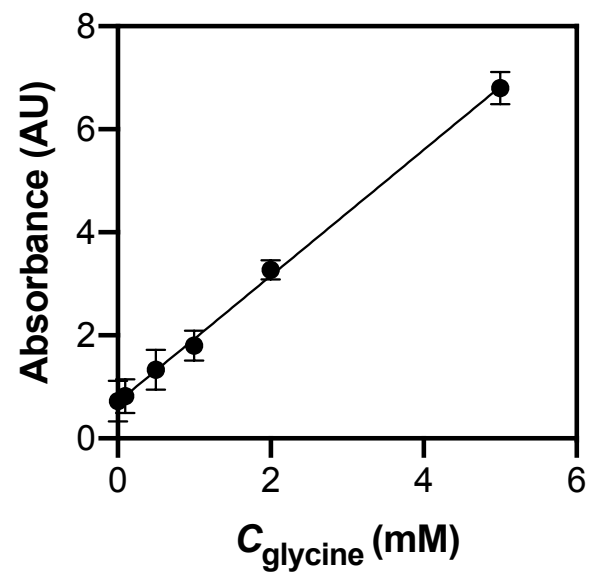

Figure S2. Calibration for TNBS assay with glycine standards. For linear regression, $\mathrm{R}^{2}=0.979$.

\section{Section S3: Dimensions of aldehyde modified CNCs}

Figure S3a shows a transmission electron microscopy (TEM) image of aldehyde-modified cellulose nanocrystals (CNCs). The histograms of CNC size distribution (based on TEM image 
analysis using ImageJ software) are shown in Figures S3b and c, respectively. The CNCs had average length and diameter of $175 \pm 50 \mathrm{~nm}$, and $20.5 \pm 3.8 \mathrm{~nm}$, respectively.

a

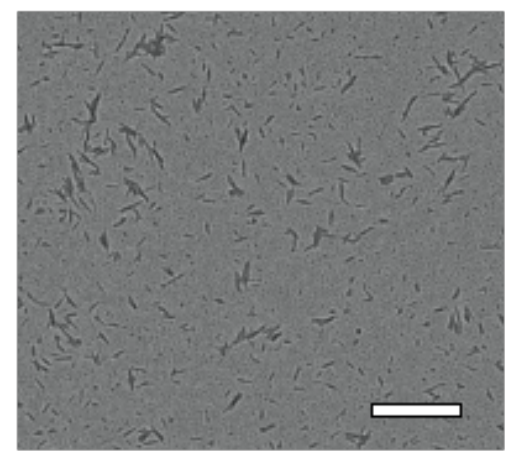

b

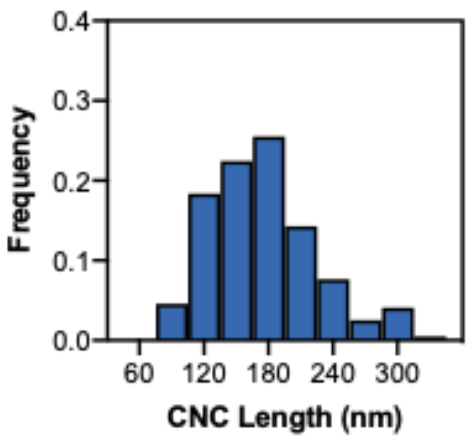

c

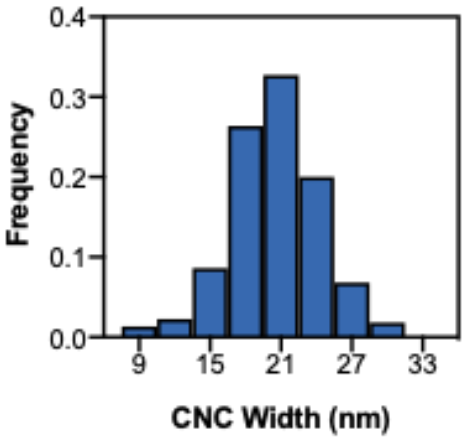

Figure S3. Aldehyde modified CNCs. (a) TEM image of aldehyde modified CNCs. Scale bar is $1 \mu \mathrm{m} .(\mathrm{b}, \mathrm{c})$ Histograms of CNC length (b) and width (c). $N=200$ in (b) and (c).

\section{Section S4: Increase in diameter and metabolic activity of breast tumor spheroids over time}

Figure S4 shows the increase in spheroid diameter and metabolic activity over time for MCF-7 and MDA-MB-231 cells. The average diameter of the MCF-7 spheroids increased over 21 days for all compositions of EKGel that were tested (Figure S4a and b). The normalized metabolic activity of MCF-7 cells also increased from day 1 to day 21 for all compositions of EKGel measured $\left(C_{\mathrm{CNC}}\right.$ from 0.3 to $2.5 \mathrm{wt} \%$ and $C_{\text {total }}$ from 1 to $5 \mathrm{wt} \%$ ). Similarly, the normalized metabolic activity of MDA-MB-231 cells increased from day 1 to day 21 for all compositions of EKGel measured ( $C_{\mathrm{CNC}}$ from 0.3 to $2.5 \mathrm{wt} \%$ and $C_{\text {total }}$ from 1 to $5 \mathrm{wt} \%$ ).

The growth in diameter of MCF-7 spheroids was decreased in stiffer and more fibrotic hydrogels. The average spheroid diameter was $109 \pm 13$ and $58 \pm 9 \mu \mathrm{m}$ in EKGel with $C_{\mathrm{CNC}}$ of 0.3 and $2.5 \mathrm{wt} \%$, respectively (two-way ANOVA, tukey's post-hoc test, $p<0.0001$ ). Spheroid diameter also decreased in stiffer hydrogels with higher $C_{\text {total }}$. More specifically, the average spheroid diameter was $95 \pm 49$ and $69 \pm 46 \mu \mathrm{m}$ in EKGel with $C_{\text {total }}$ of 1.0 and $5.0 \mathrm{wt} \%$, respectively (Figure 
S4a, two-way ANOVA, tukey's post-hoc test, $p<0.0001$ ). The impact of EKGel composition on metabolic activity is described in the Main Text.
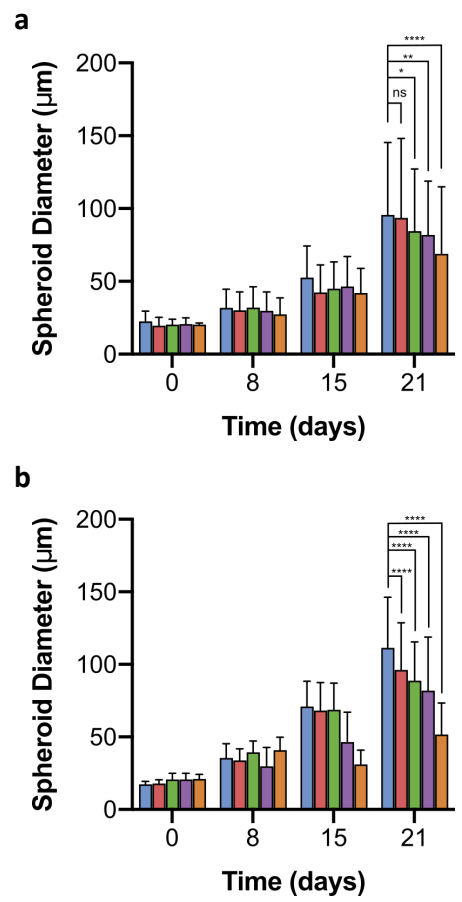

c

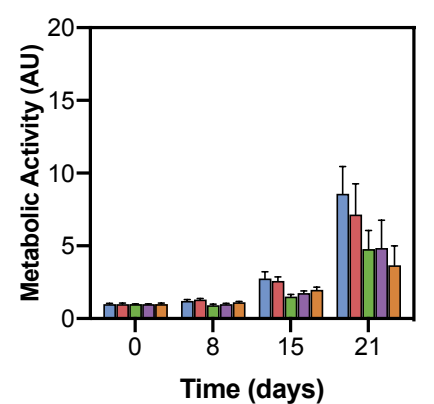

d

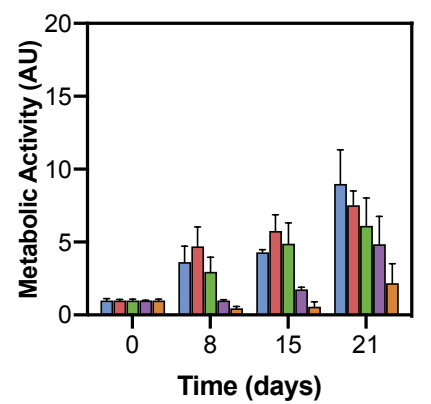

e

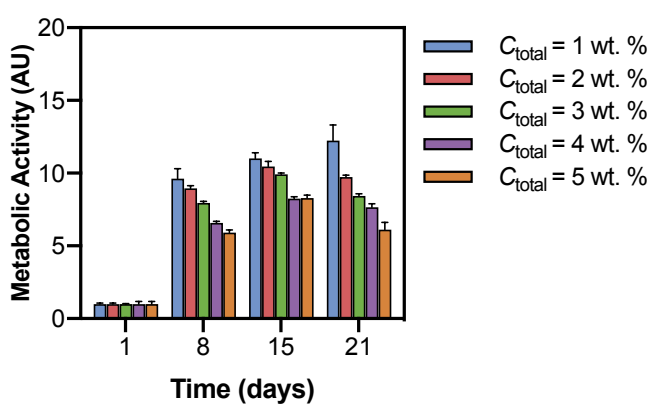

f

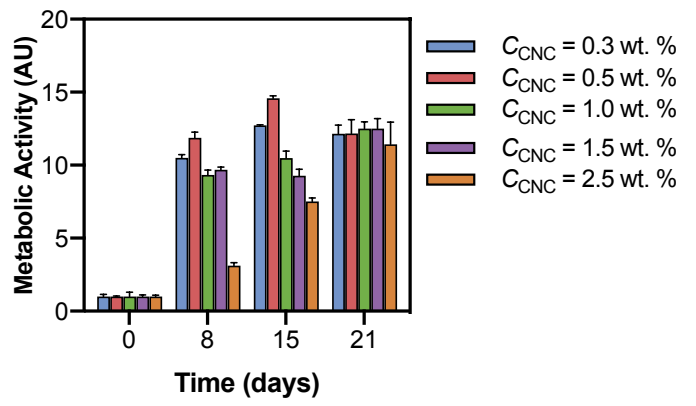

Figure S4. Growth of MCF-7 and MDA-MB-231 spheroids in EKGel over 21 days. All data is shown as mean \pm st. dev. $(a, b)$ Variation in MCF-7 spheroid diameter with $C_{\mathrm{CNC}}(\mathrm{a})$ and with varying $C_{\text {total }}$ at $C_{\mathrm{CNC}} / C_{\text {gel }}=0.5$ (b). For each bar in (a, b) $N=300$ spheroids. (c, d) Variation in the normalized metabolic activity of MCF-7 spheroids grown in EKGel with varying $C_{\mathrm{CNC}}(\mathrm{c})$ and with varying $C_{\text {total }}$ at $C_{\mathrm{CNC}} / C_{\mathrm{gel}}=0.5(\mathrm{~d})$. (e, f) Variation in the normalized metabolic activity of MDA-MB-231 spheroids grown in EKGel with varying $C_{\mathrm{CNC}}$ (e) and with varying $C_{\text {total }}$ at $C_{\mathrm{CNC}} / C_{\mathrm{gel}}=0.5$ (f). Two-way ANOVA tukey's post-hoc test: ${ }^{\mathrm{ns}} p>0.1,{ }^{*} p<0.1,{ }^{* *} p<0.01$, ${ }^{* * * *} p<0.0001$. Statistical information for (c-f) is displayed in Figure 5, Main Text.

\section{Section S5: Influence of gelatin concentration on the growth of MCF-7 spheroids}

In order to investigate the influence of gelatin concentration on the growth of MCF-7 spheroids in EKGel, we varied $C_{\text {gel }}$ while $C_{\mathrm{CNC}}$ was kept constant at $1 \mathrm{wt} \%$ (Figure $\mathrm{S} 5$ ). Notably, when $C_{\mathrm{gel}}$ was less the $1.5 \mathrm{wt} \%$, the diameter of the MCF-7 spheroids after 21 days of culture was significantly lower than when $C_{\text {gel }}>1.5 \mathrm{wt} \%\left(22 \pm 7 \mu \mathrm{m}\right.$ when $C_{\mathrm{gel}}=0.5 \mathrm{wt} \%$, two-way ANOVA tukey's post-hoc test, $p<0.0001$, and $46 \pm 25 \mu \mathrm{m}$, two-way ANOVA tukey's post-hoc test, 
$p<0.0001)$. The diameter of MCF-7 spheroids after 21 days of culture then plateaued from 1.5 to 3.0 wt. $\%$ ( $85 \pm 33 \mu \mathrm{m}$ to $87 \pm 30 \mu \mathrm{m}$, two-way ANOVA tukey's post-hoc test, $p=0.7)$. This indicates that there are insufficient cell adhesion ligands to support the growth of MCF-7 spheroids in EKGel with $C_{\mathrm{gel}}<1.5 \mathrm{wt} \%$.

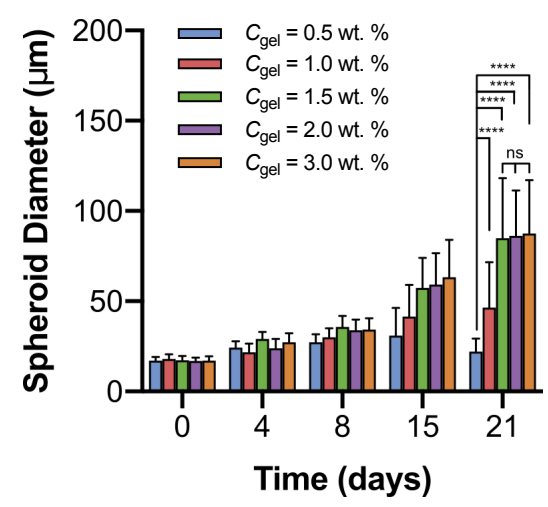

Figure S4. Growth of MCF-7 spheroids in EKGel with varying $C_{\mathrm{gel}}$ and $C_{\mathrm{CNC}}=1 \mathrm{wt} \%$. All data are shown as mean \pm st. dev. Two-way ANOVA tukey's post-hoc test: ${ }^{\mathrm{ns}} p>0.1,{ }^{* * * *} p<0.0001$.

\section{References:}

(1) Prince, E.; Alizadehgiashi, M.; Campbell, M.; Khuu, N.; Albulescu, A.; De France, K.; Ratkov, D.; Li, Y.; Hoare, T.; Kumacheva, E. Patterning of Structurally Anisotropic Composite Hydrogel Sheets. Biomacromolecules 2018, 19 (4), 1276-1284. 